\title{
预测铝合金单轴力学性能的复合型双锥压入法
}

\author{
陈 辉 ${ }^{1,2}$ 蔡力勋 $^{3}$ 彭 晖 ${ }^{1,2}$ \\ (1. 长沙理工大学土木工程学院 长沙 410114; \\ 2. 长沙理工大学桥梁工程安全控制教育部重点实验室 长沙 410114; \\ 3. 西南交通大学力学与工程学院 成都 610031)
}

\begin{abstract}
摘要: 雉形压入作为一种发展较早的压入测试手段, 可在一定范围内实现材料单轴力学性能的唯一获取, 但目前仍存在稳定 性和简便性等方面的缺陷。为避免传统雉形压入需要两个或以上压头分别压入不同位置且压点之间存在匹配性等问题, 创新 地设计和使用了复合型双锥压头。基于能量等效原理建立了雉形压入的弹塑性加载模型, 并通过较大范围的数值计算验证了 该模型的准确性。通过关联复合型双雉压入与单雉压入的响应曲线, 发展了一种复合型双雉压入方法(Composite dual-conical indentation method, CDIM)。针对 3 种铝合金, 利用复合型压入试验获得了稳定的载荷-深度曲线, 并结合 CDIM 预测了材料 的单轴应力-应变曲线。结果表明, 复合型压入预测结果与单轴拉伸结果较为一致。
\end{abstract}

关键词: 复合型双锥压入; 能量等效原理; 有限元分析; 力学性能

中图分类号: $\mathrm{O} 348$

\section{Composite Dual-conical Indentation Method for Predicting the Uniaxial Mechanical Properties of Aluminum Alloys}

\author{
CHEN Hui ${ }^{1,2}$ CAI Lixun ${ }^{3}$ PENG Hui ${ }^{1,2}$
}

(1. School of Civil Engineering, Changsha University of Science \& Technology, Changsha 410114;

2. Key Laboratory of Bridge Engineering Safety Control by Department of Education,

Changsha University of Science \& Technology, Changsha 410114;

3. School of Mechanics and Engineering, Southwest Jiaotong University, Chengdu 610031)

\begin{abstract}
Conical indentation, as an early developed indentation test method, can uniquely acquire the uniaxial mechanical properties of materials within a certain range, but there are still defects in stability and simplicity. In order to avoid the problems that the traditional dual-conical indentation requires two indenters to penetrate different positions respectively and the compatibility between the indentation points, a composite dual-conical indenter is innovatively designed and used. Based on equivalent energy principle, an elastic-plastic loading model for conical indentation is established and its accuracy is verified via a large range of numerical simulations. By correlating response curves of composite dual-conical indentation and single-conical indentation, a composite dual-conical indentation method(CDIM) is developed. For three kinds of aluminum alloys, the stable load-depth curves are obtained by the composite indentation tests, and the uniaxial stress-strain curves are predicted by CDIM. The results show that the predictions of CDIM are consistent with those from uniaxial tension.
\end{abstract}

Key words: composite dual-conical indentation; equivalent energy principle; finite element analysis; mechanical properties

\section{0 前言}

材料单轴力学性能是对材料或结构进行力学分

* 国家自然科学基金 $(51878068,11872320$ )、湖南省教育厅优秀青年 (20B028)和教育部重点实验室开放(19KB04)资助项目。20200429 收到 初稿, 20210706 收到修改稿
析与设计的重要基础, 也是实现工程构件安全评价 的前提条件。一般地, 获取材料弹塑性力学性能的 传统方法是从工程构件或母材上割取具有一定尺寸 的标准试样后在实验室进行拉伸或压缩试验。然而 随着结构趋于小型化、精细化(如微机电系统等), 传统拉伸等方法易受构件尺度限制, 难以取样并有 
效地展开试验。并且, 对于焊接结构或常规在役结 构, 采用传统方法要么难以获得不同区域(焊缝区、 热影响区等)的力学性能, 要么在截取试样时须损害 结构服役状态。此外, 对于贵重的新兴材料(如结构 纳米材料等)及金属材料(如锆合金、银合金等), 传 统方法易产生成本过高和回收能耗大等问题。针对 上述情况, 近三十年来, 压入测试方法快速发展并 成为材料力学测试研究的热点。由于载荷-压深满足 二次幂律特征(即 Kick 定律)、金刚石圆雉压头易于 高精度加工等优势, 圆雉压入测试方法得到了较多 的研究和发展。通过雉形压入获取材料弹塑性参数 的方法主要有三类: 代表性应变法、数值方程法和 智能优化算法。

代表性应变法的核心思想在于通过简单关联压 入特征变形、接触应力与单轴拉伸应变、应力, 来代 表性地表征材料的应力应变关系。BUCAILLE 等 ${ }^{[1]}$ 在 DAO 等 ${ }^{[2]}$ 的研究基础上分别给出了的代表性应 变与半雉角余切值的比例关系及代表性应力与加载 曲率的拟合关系, 结合两种不同角度雉压入测得的 加载曲率实现了材料塑性参数的获取。但建立不受 硬化指数影响的代表性应力-加载曲率关系需要基 于不同代表性应变值进行多次的多参数组合下的有 限元(Finite element analysis, FEA)计算, 并且该方法 依赖复杂的迭代求解。之后, CAO 等 ${ }^{[3]}$ 和 OGASAWARA 等 ${ }^{[4]}$ 分别定义了不同的多项式型代 表性应变, 事实上雉压入的代表性应变不仅与压头 角度有关, 还受材料的屈服强度与硬化程度的影 响 $^{[5-6]}$ 。

数值方程法, 即结合量纲分析采用大量参数化 有限元计算结果建立压入测试参数, 如加载曲率、 加载功等与材料性能参数之间的数值回归方程, 然 后代入实测参数求解材料参数的方法。 $\mathrm{THO}$ 等 ${ }^{[7]}$ 在 硬化指数 $n \in[0.1,0.6]$, 联合模量-屈服应力比 $E^{*} / \sigma_{\mathrm{y}}$ $\in[60,1000]$ 的范围内进行有限元计算, 通过 500 余 次有限元计算结果和量纲分析分别建立了无量纲塑 性功、无量纲卸载刚度与材料弹塑性参数之间的关 系。姚博等 ${ }^{[8]}$ 基于压入加卸载曲线的数值模拟建立 了不同硬化指数下材料名义硬度、加卸载弹塑性功 之比与材料屈服应变之间的线性关系, 但该方法仍 依赖大量有限元计算确定回归参数, 缺乏足够理论 基础。PÖHL ${ }^{[9]}$ 独特地选用半雉角为 $75.8^{\circ}$ 和 $70.3^{\circ}$ 的两种圆雉压头, 分别采用数值模拟获得了用压入 功或加载曲率与硬化指数表示的材料强化系数表达 式, 在一定程度上实现了材料塑性参数的唯一识别。 石新正等 ${ }^{[10]}$ 试图通过 Vickers 压头(等效于 $70.3^{\circ}$ 圆
雉)仪器化压入聚集式(一体化)地测量材料的弹性模 量、硬化指数与强度, 但该方法需进行三次特定位 置的压入加卸载才可得到三个关于材料参数和压入 响应参数的无量纲等式。

智能优化算法是指通过数学优化算法在预先或 实时建立的样本库中迭代 “寻找” 出与压入试验曲 线最匹配的材料应力-应变关系。PEYROT 等 ${ }^{[11]}$ 在采 用 BUCAILLE 等 ${ }^{[1]}$ 的雉形压入模型预测近似值作为 初始值, 进而通过发展随机优化算法借助商用求解 软件的迭代计算实现了材料力学性能的更精准获 取。HAJ-ALI 等 ${ }^{[12]}$ 利用神经网络算法和有限元模拟 结合的方式对纳米晶铜薄膜-硅基体系统进行了弹 塑性力学性能纳米压入预测研究, 但预测精度有限。 陈辉等 ${ }^{[13]}$ 结合数值方程采用了一种基于双雉形压 入离散载荷值的比例缩放式迭代算法, 获取了核 电钛合金的单轴应力应变关系。HOSSEINZADEH 等 ${ }^{[14]}$ 采用遗传算法对材料力学性能参数进行压入 测试研究, 并针对不锈钢材料进行了验证与应用。 但此类优化方法犹如 “黑匣子” 无法揭示压入弹塑 性力学问题的内在关系和物理过程。

本文基于能量等效原理 ${ }^{[15-16]}$, 将雉形压入功表 达成积分中值点应变能密度与有效变形体积之积, 再结合空腔模型对中值点应变和变形域体积进行合 理假设, 导出了不同角度下、HOLLOMON 幂律材 料的载荷-深度响应与材料参数之间的半解析模型。 经过针对模量为 $70 \mathrm{GPa}$ 时的若干次有限元计算确 定了适用于铝合金材料的模型参数。通过关联复合 型双雉压入曲线同与两种角度单一圆雉压入曲线, 提出了复合型双雉压入新方法。最终采用 3 种实际 铝合金材料对所提方法进行了验证和应用。

\section{1 雉形压入理论模型}

\section{1 弹性卸载模型}

对于常用铝合金材料, 其弹塑性应力-应变关系 通常近似满足各向同性、率无关的 $J_{2}$ 流动应力准则, 且可近似表达为分段形式的 HOLLOMON 幂律模 型, 即

$$
\sigma= \begin{cases}E \varepsilon & \varepsilon \leqslant \varepsilon_{\mathrm{y}} \\ E^{n} \sigma_{\mathrm{y}}^{1-n} \varepsilon^{n} & \varepsilon \geqslant \varepsilon_{\mathrm{y}}\end{cases}
$$

式中, $\sigma$ 表示应力, $\varepsilon$ 表示应变, $E$ 为弹性模量, $\sigma_{\mathrm{y}}$ 为名义屈服应力, $\varepsilon_{\mathrm{y}}$ 为名义屈服应变, $n$ 为应变硬 化指数。鉴于材料弹性阶段和弹塑性阶段的本构关 系有明显差异, 而弹性加载阶段难以准确测量, 即 
便有 $\mathrm{SNEDDON}^{[17]}$ 的理论解也难以应用于弹性参数 测试。因此, 通过雉形压入获取材料弹性模型一般 通过卸载折合模量 $E_{\mathrm{r}}$ 得到, 即

$$
E=\left(1-v^{2}\right) /\left[1 / E_{\mathrm{r}}-\left(1-v_{\mathrm{i}}^{2}\right) / E_{\mathrm{i}}\right]
$$

式中, $E$ 与 $v$ 分别为被压材料弹性模量和泊松比, $E_{\mathrm{i}}$ 和 $v_{\mathrm{i}}$ 分别为压头弹性模量和泊松比。而折合模量 $E_{\mathrm{r}}$ 可视为将压头与被压材料串联后形成系统的联合模 量, 可通过卸载刚度得到

$$
E_{\mathrm{r}}=\frac{\sqrt{\pi} S}{2 \beta \sqrt{A_{\mathrm{c}}}}
$$

式中, $\beta$ 为压头系数, $A_{\mathrm{c}}$ 为压痕投影面积, $S$ 为卸载 刚度, 即卸载初始阶段的斜率。一般先采用如下函 数按最小二乘法拟合卸载曲线顶部 $25 \%$ \% $50 \%$ 的部 分数据

$$
P=\alpha\left(h-h_{\mathrm{r}}\right)^{t}
$$

式中, $\alpha$ 和 $t$ 是拟合系数和拟合指数, $h_{\mathrm{r}}$ 是卸载后残 余深度。然后对式(4)在卸载点 $h_{\mathrm{m}}$ 处进行求导得卸 载刚度为

$$
S=\left.\frac{\mathrm{d} P}{\mathrm{~d} h}\right|_{h=h_{\mathrm{m}}}=\alpha t\left(h_{\mathrm{m}}-h_{\mathrm{r}}\right)^{t-1}
$$

对于雉形压入, 压痕投影面积 $A_{\mathrm{c}}$ 定义为

$$
A_{\mathrm{c}}=\pi h_{\mathrm{c}}^{2} \tan ^{2} \theta
$$

式中, $h_{\mathrm{c}}$ 为有效接触深度, 常采用介于残余深度 $h_{\mathrm{r}}$ 和卸载点深度 $h_{\mathrm{m}}$ 间的经验公式估算，即

$$
h_{\mathrm{c}}=h_{\max }-\gamma \frac{P_{\max }}{S}
$$

式中, $\gamma$ 为压头形状相关常数, 圆雉压入时 $\gamma$ 取为 0.72 。总之, 通过压入卸载曲线获得卸载刚度 $S$ 和 接触面积 $A_{\mathrm{c}}$ 后, 代入式(3)求得折合模量, 进而按照 式(2)可求出被压材料弹性模量 $E$, 该方法即为经典 的 OLIVER-PHARR 方法 ${ }^{[18-19]}$ 。

\section{2 弹塑性加载模型}

对于弹塑性雉形压入, 在连续的变形域中必存 在积分中值点 $M\left(x_{M}, y_{M}, z_{M}\right)$, 使得在该点的材料代 表性体积单元 $(\mathrm{RVE})$ 的应变能密度 $u_{M}$ 与有效变形域 $\Omega$ 的体积 $V$ 的乘积等于雉压入载荷作用下的形变能 $U$ 。根据积分中值定理

$$
U=u_{M} V
$$

根据 von-Mises 等效, 处于复杂应力状态的 $M$ 点材料 RVE 的应变能密度可等效转化为简单应力 状态(单轴应力状态)下 RVE 的能量密度, 即

$$
u_{M}=\int_{0}^{\varepsilon_{\mathrm{ij}}} \sigma_{\mathrm{ij}} \mathrm{d} \varepsilon_{\mathrm{ij}}=\int_{0}^{\varepsilon_{\mathrm{eq}}} \sigma_{\mathrm{eq}} \mathrm{d} \varepsilon_{\mathrm{eq}}
$$

根据式(1)和(9)可得初始弹性应变能密度

$$
u_{\mathrm{e}}=\int_{0}^{\varepsilon_{\mathrm{y}}} E \varepsilon \mathrm{d} \varepsilon=\frac{E \varepsilon_{\mathrm{y}}^{2}}{2}=\frac{K \varepsilon_{\mathrm{y}}^{1+n}}{2}
$$

及弹塑性应变能密度

$$
u_{\text {ep }}=\int_{\varepsilon_{\mathrm{y}}}^{\varepsilon_{\mathrm{eq}}} K \varepsilon^{n} \mathrm{~d} \varepsilon=\frac{K}{1+n}\left(\varepsilon_{\mathrm{eqM}}^{1+n}-\varepsilon_{\mathrm{y}}^{1+n}\right)
$$

综合式(10)与(11)可得 $M$ 点应变能密度

$$
u_{\mathrm{eq}}=u_{\mathrm{e}}+u_{\mathrm{ep}}=\frac{K}{1+n}\left(\varepsilon_{\mathrm{eqM}}^{1+n}-\frac{1-n}{2} \varepsilon_{\mathrm{y}}^{1+n}\right)
$$

式中, $\varepsilon_{\mathrm{eqM}}$ 为中值点 $M$ 处 RVE 的等效应变。考虑到 $\varepsilon_{\mathrm{y}}$ 相比于 $\varepsilon_{\mathrm{eqM}}$ 足够小，即

$$
\frac{1-n}{2}\left(\frac{\varepsilon_{\mathrm{y}}}{\varepsilon_{\mathrm{eqM}}}\right)^{1+n} \ll 1
$$

$M$ 点应变能密度可近似表达为

$$
\left.u_{M}\right|_{\left(x_{M}, y_{M}, z_{M}\right)}=\frac{E^{n} \sigma_{\mathrm{y}}^{1-n} \varepsilon_{\mathrm{eqM}}^{1+n}}{1+n}
$$

因此结合式(8)与式(14)可得总形变能为

$$
U=\left.\frac{E^{n} \sigma_{\mathrm{y}}^{1-n} V}{1+n} \varepsilon_{\text {eqM }}^{1+n}\right|_{\left(x_{M}, y_{M}, z_{M}\right)}
$$

JOHNSON 根据 SAMUALS 对压入样品剖面的 金相观察提出了经典的空腔模型 ${ }^{[20]}$, 该模型将钝形 压入弹塑性变形域简化为放射状的半球形，且同一 球面上的应变互等，因此依据体积不变假定可得圆 雉形压入的有效变形域体积为

$$
V=\frac{2 \pi}{3} c^{3}=\frac{2 \pi}{3}\left(\alpha_{1} h \tan \theta\right)^{3}=k_{1} h^{3} \tan ^{3} \theta
$$

式中, $c$ 为有效变形域的半径, $k_{1}$ 为有效体积系数 且 $k_{1}=2 \pi \alpha_{1}{ }^{3} / 3$ 。类比于 TABOR 提出的雉压入代表性 应变为 $0.2 \cot \theta$, 可假定积分中值点 $M$ 的应变为

$$
\varepsilon_{\mathrm{eq} M}=k_{2} \cot \theta
$$

式中, $k_{2}$ 为中值点应变系数。结合式(15)、式(16) 和式(17)可得变形能表达式为

$$
U=\frac{E^{n} \sigma_{\mathrm{y}}^{1-n} k_{1} k_{2}^{1+n} \tan ^{2-n} \theta}{1+n} h^{3}
$$

结合功能原理, 通过求导可得压入载荷-深度关系为

$$
P=\frac{3 E^{n} \sigma_{\mathrm{y}}^{1-n} k_{1} k_{2}^{1+n} \tan ^{2-n} \theta}{1+n} h^{2}
$$

根据雉形压入近似满足的 $\mathrm{KICK}$ 定律, 有 


$$
P=C h^{2}
$$

选取常用的半雉角分别为 $\theta_{1}$ 和 $\theta_{2}$ 的两种针雉压 头, 可得如下方程组

$$
\left\{\begin{array}{l}
C_{\theta_{1}}=\frac{3 E^{n} \sigma_{\mathrm{y}}^{1-n} k_{1 \_} \theta_{1} k_{2 \_} \theta_{1}^{1+n} \tan ^{2-n} \theta_{1}}{1+n} \\
C_{\theta_{2}}=\frac{3 E^{n} \sigma_{\mathrm{y}}^{1-n} k_{1-\theta_{2}} k_{2_{-} \theta_{2}}{ }^{1+n} \tan ^{2-n} \theta_{2}}{1+n}
\end{array}\right.
$$

在通过弹性卸载模型获得被压材料弹性模量 $E$ 后，通过求解方程式(21)得材料塑性参数为

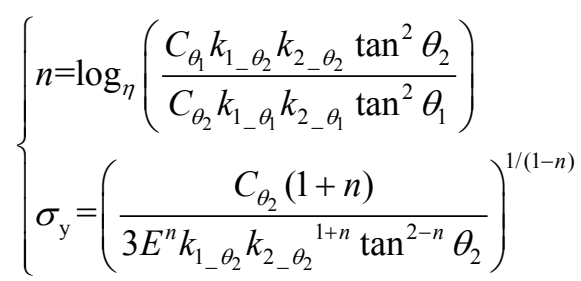

式中, 常量底数 $\eta=k_{2 \_} \theta_{1} \tan \theta_{2} /\left(k_{2 \_} \theta_{2} \tan \theta_{1}\right)$ 。

\section{2 弹塑性模型的确立与验证}

\section{1 模型参数确定方法}

本文借助 ANSYS14.5 商业有限元软件确定了 两种常用圆雉压头 (即 $\theta$ 分别为 $70.3^{\circ}$ 和 $60^{\circ}$ ) 下的 待定模型参数 $k_{1}$ 和 $k_{2}$ 。如图 1 所示, 压入有限元模 型为二维轴对称模型, 被压材料采用二维单元 plane182 单元进行划分, 压头和被压材料表面分别 设置为 rigid169 和 contact172 单元。为了确保计算 收玫于准确结果, 被压区域经过特别密化。模型底 部为坚直方向约束 $Y=0$, 左侧为轴对称约束。压头 半雉角为 $60^{\circ}$, 材料弹性模量为 $70 \mathrm{GPa}$, 泊松比 $v$ $v=0.33$ 。考虑到摩擦对相当范围内的压入曲线影响 微弱 ${ }^{[21]}$, 不妨假定压头与材料接触面摩擦满足经典 的库仑摩擦模型, 摩擦因数为 0.15 。

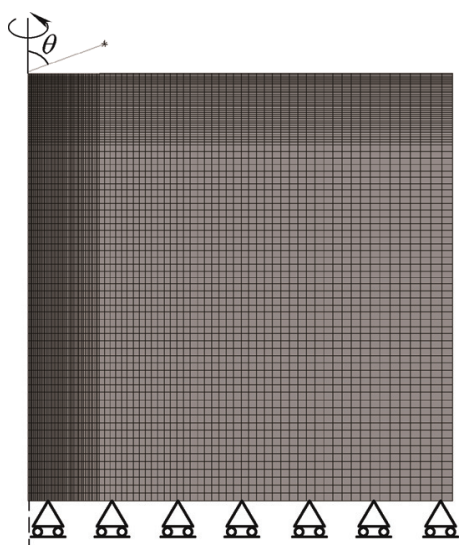

图 1 雉形压入有限元模型
为了防止压入接触的局部网格密度对计算结果 可能造成的影响, 将不同网格密度下的载荷-深度曲 线进行比较以确定适当的收敛网格尺寸。定义最小 网格尺寸 $l_{\min }$ 等于 $1 \mu \mathrm{m}$ 时为 1 倍网格密度, $l_{\min }$ 是 $0.5 \mu \mathrm{m}$ 时为 2 倍网格密度, 以此类推。图 2 给出了 网格密度对压入 $P-h$ 曲线的影响, 结果表明采用 1 倍网格密度时已经满足计算要求, 但存在小幅度的 波动, 为了更好地兼顾精度和效率, 本文采用 2 倍 网格密度进行有限元分析。

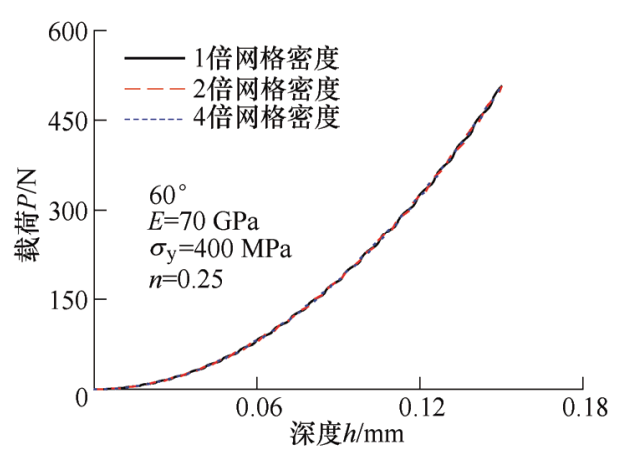

图 2 网格密度对雉形压入曲线的影响

为了确定 $70.3^{\circ}$ 和 $60^{\circ}$ 雉角下的 $k_{1}$ 和 $k_{2}$ 的值, 分别在固定的弹性模量 $E$ 和屈服应力 $\sigma_{\mathrm{y}}$ 下, 针 对 $n$ 在 $0.1 \sim 0.4$ 的范围内以 0.1 为步长的变化, 进 行了压入载荷-深度曲线模拟, 采用三次多项式平滑 处理后的模拟曲线如图 3 所示。若直接通过幂律拟 合加载曲线得到加载曲率 $C$ 值, 常常会由于幂指数 在 1.99 2.01 范围的变化而产生上下浮动, 为了精 准获取 Kick 律特征参数 $C$, 可将图 3 所示 $P \sim h$ 数 据转化成 $P^{1 / 2} \sim h$ 关系, 然后进行正比例函数拟合, 即可得到 $C$ 值(图 4)。

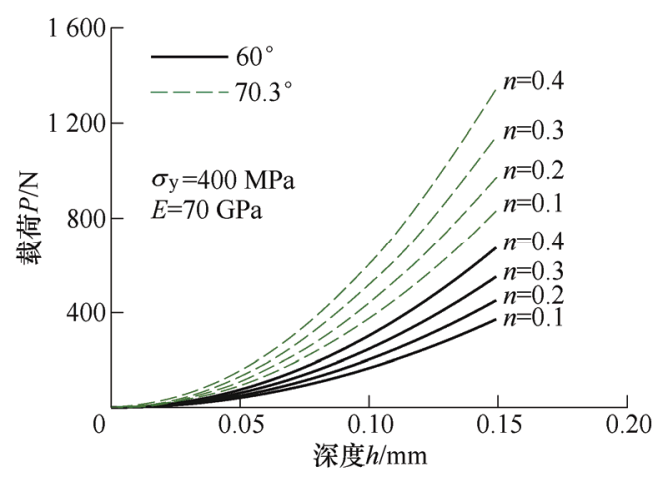

图 3 参考屈服应力下的 $P \sim h$ 计算曲线

基于图 4 方式获取的加载曲率 $C$, 图 5 给出了 弹性模量为 $70 \mathrm{GPa}$ 和屈服应力为 $400 \mathrm{MPa}$ 时, $C$ 值 随着硬化指数 $n$ 的变化关系, 并进一步回归即可得 到 $k_{1}$ 和 $k_{2}$ 。 


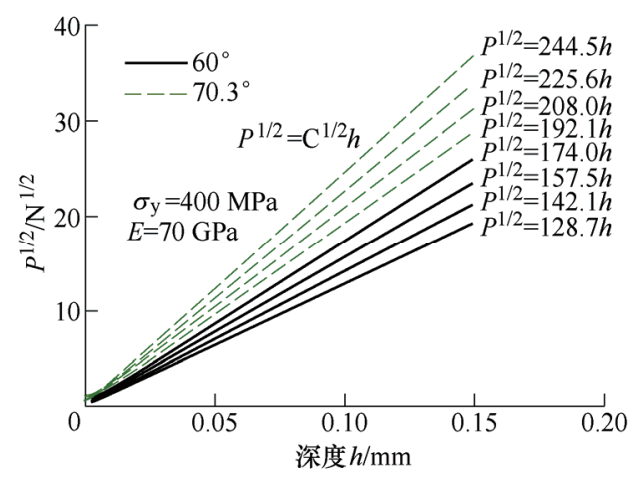

图 4 用于确定 $C$ 的 $P^{1 / 2} \sim h$ 比例关系

然而由于据图 5 所得 $k_{1}$ 和 $k_{2}$ 只考虑了硬化指数 $n$ 的影响, 为了保证两种角度下所得 $k_{1}$ 和 $k_{2}$ 在其他 屈服应力条件下仍然具有较高的预测精度, 可引入 $k_{1}$ 与屈服应力比之间的关系为

$$
k_{1-\text { cor }}=m_{1}\left(\frac{\sigma_{\mathrm{y}}}{\sigma_{\mathrm{y}}^{*}}\right)^{m_{2}} k_{1}
$$

式中, $m_{1}$ 和 $m_{2}$ 分别为修正系数和修正指数, $\sigma_{\mathrm{y}}{ }^{*}$ 为参考 屈服应力且取值为 $400 \mathrm{MPa}$ 。为了确定 $m_{1}$ 和 $m_{2}$, 不妨 将弹性模量 $E$ 和硬化指数 $n$ 分别固定为 $70 \mathrm{GPa}$ 和 0.2 , 分别计算屈服应力 $\sigma_{\mathrm{y}}$ 为 $100 \mathrm{MPa} 、 200 \mathrm{MPa} 、 400 \mathrm{MPa}$ 和 $600 \mathrm{MPa}$ 时的加载曲率与预测曲率之比, 即可得到 $m_{1}$ 和 $m_{2}$ 的值列于表 1 。而对于中值点应变系数 $k_{2}$, 其 受屈服应力影响较小 ${ }^{[15]}$, 可近似看作常数(表 1)。

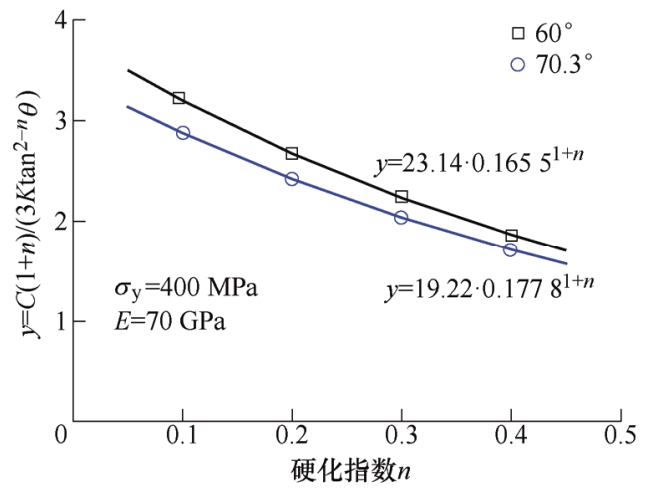

图 5 用于确定 $k_{1}$ 和 $k_{2}$ 的 $y \sim n$ 幂律关系

表 1 无量纲量 $\boldsymbol{m}_{1} 、 \boldsymbol{m}_{2}$ 及 $\boldsymbol{k}_{2}$ 的值

\begin{tabular}{cccc}
\hline 角度 $\theta /\left(^{\circ}\right)$ & 系数 $m_{1}$ & 指数 $m_{2}$ & 系数 $k_{2}$ \\
\hline 60 & 1.192 & 0.8522 & 0.1655 \\
70.3 & 1.256 & 0.8122 & 0.1778 \\
\hline
\end{tabular}

至此, 针对铝合金材料的雉压入弹塑性模型及 其待定参数已经完整确立, 如式(19)、式(22)及表 1

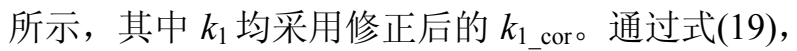
若已知铝合金材料 HOLLOMON 模型参数, 可以直 接预测其压入载荷-深度响应曲线, 称为 “正向预
测”; 反之, 若已知材料压入响应曲线, 在弹性模量 已知或者已根据 OLIVER-PHARR 方法获得的基础 上, 可按照图 4 的方法得到加载曲率 $C$, 代入式(22) 得到材料的屈服应力与硬化指数, 称为 “反向预测”。

\section{2 数值结果验证}

为验证所提弹塑性压入模型在铝及其合金材料 范围内的准确性, 首先考察了弹性模量 $E$ 对模型预 测结果的影响, 从图 6 可以看出当弹性模量在 65 $80 \mathrm{GPa}$ 范围变化时, 模型仍然适用。

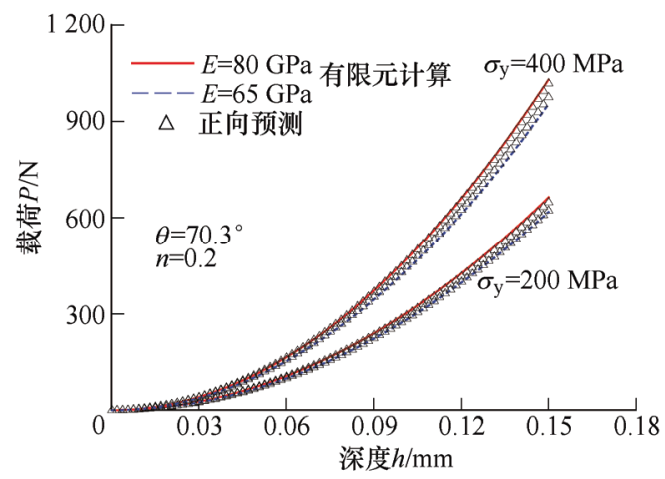

图 6 弹性模量对模型预测的影响

进一步地, 图 7a 7d 给出了模型预测载荷-深 度曲线与有限元计算结果进行对比, 结果表明: 当 材料屈服应力 $\sigma_{\mathrm{y}} \in[100,600] \mathrm{MPa}$ 、硬化指数 $n \in[0.1$, 0.4]时, 式(19)正向预测结果与有限元计算结果吻合 良好。其中正向预测的载荷-深度曲线只在压头为 $70.3^{\circ}$ 、材料参数为 $\sigma_{\mathrm{y}}=600 \mathrm{MPa}$ 且 $n \geqslant 0.3$ 时误差在 $2 \%$ 以上, 其余均为 $1 \%$ 以内。

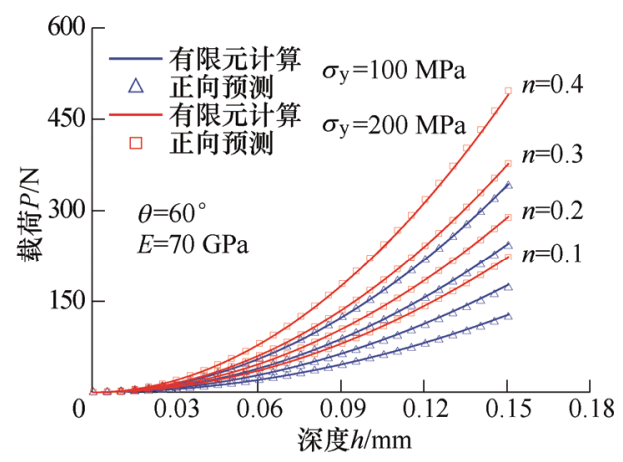

(a) $\sigma_{\mathrm{y}}$ 取 $100 \mathrm{MPa}$ 与 $200 \mathrm{MPa}, \theta=60^{\circ}$

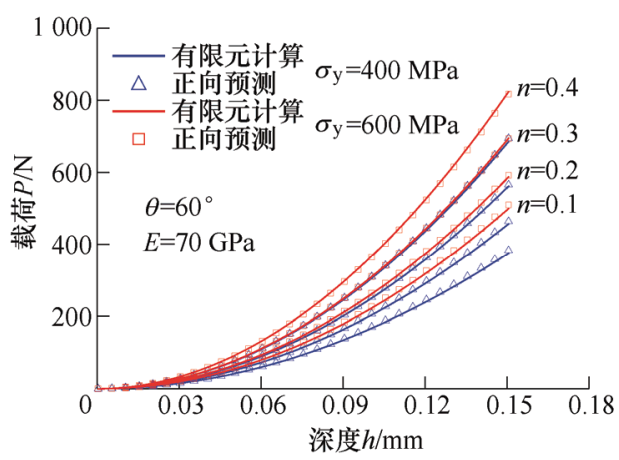

(b) $\sigma_{\mathrm{y}}$ 取 $400 \mathrm{MPa}$ 与 $600 \mathrm{MPa}, \theta=60^{\circ}$ 


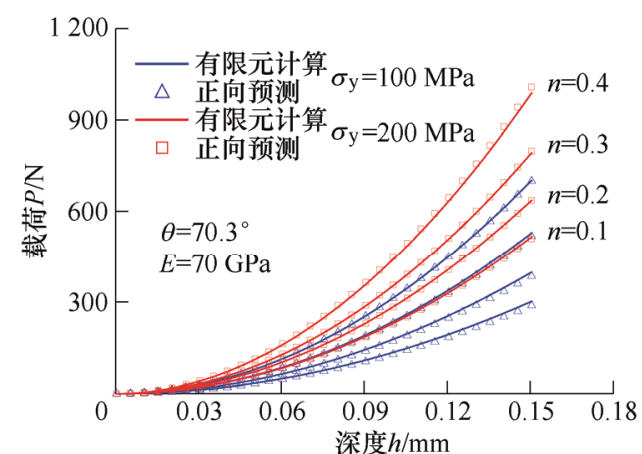

(c) $\sigma_{\mathrm{y}}$ 取 $100 \mathrm{MPa}$ 与 $200 \mathrm{MPa}, \theta=70.3^{\circ}$

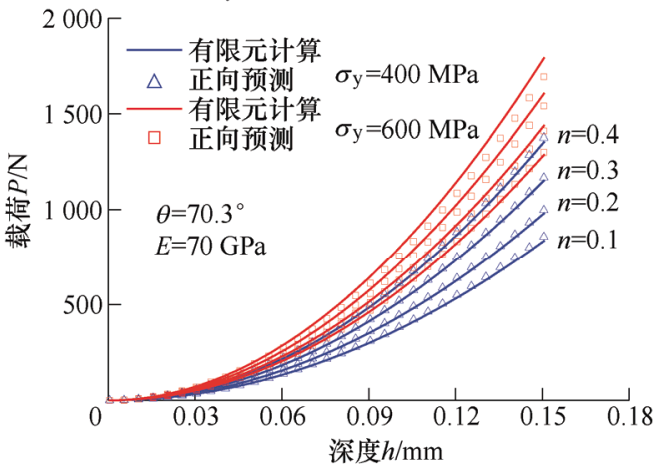

(d) $\sigma_{\mathrm{y}}$ 取 $400 \mathrm{MPa}$ 与 $600 \mathrm{MPa}, \theta=70.3^{\circ}$

图 7 不同材料的压入模型预测曲线与 FEA 结果对比

为验证雉压模型在预测铝合金材料单轴力学性 能时的可用性和有效性, 图 8 给出了有限元计算输 入的应力-应变关系同反向预测得到的应力-应变曲 线之间的对比。结果显示, 二者在较大的应变范围 内保持良好的一致性。

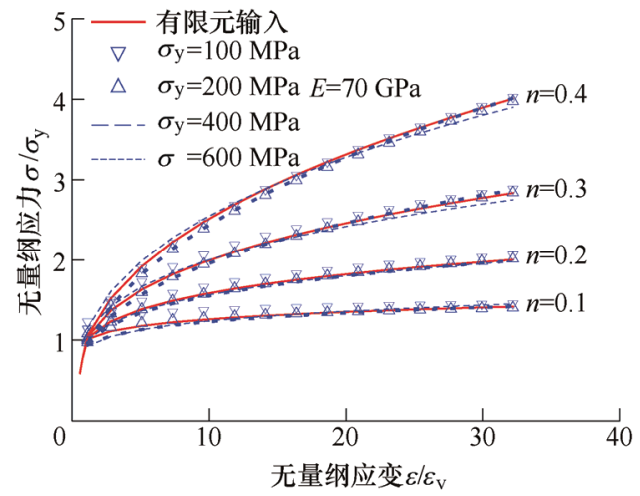

图 8 不同材料压入反向预测与 FEA 输入对比

\section{3 复合型双雉压入方法}

由于已有的雉形压入方法通常需两个或两个以 上压头分别压入不同位置, 且不同压点测量结果之 间常存在能否稳定匹配等问题。事实上, 我们发现 可以通过有机地组合两种雉角于一体实现复合型压 入, 从而解决现存的问题。设计下段雉角为 $70.3^{\circ}$ 而上段雉角为 $60^{\circ}$ 的复合型双雉压头, 下段圆雉高 度 $H$ 为 $80 \mu \mathrm{m}$, 采用与图 1 类似的网格模型, 只将
代表压头的刚性线更换成包含两种角度的两段刚性 线, 有限元计算结果显示压入响应呈现典型的两阶段 加载曲线(图 9, 以 $\sigma_{\mathrm{y}}=400 \mathrm{MPa}$ 和 $n=0.2$ 为例), 第一 段即为 $70.3^{\circ}$ 单雉压入曲线; 而第二段曲线通过平移 至满足 Kick 定律时略高于 $60^{\circ}$ 单雉压入曲线。

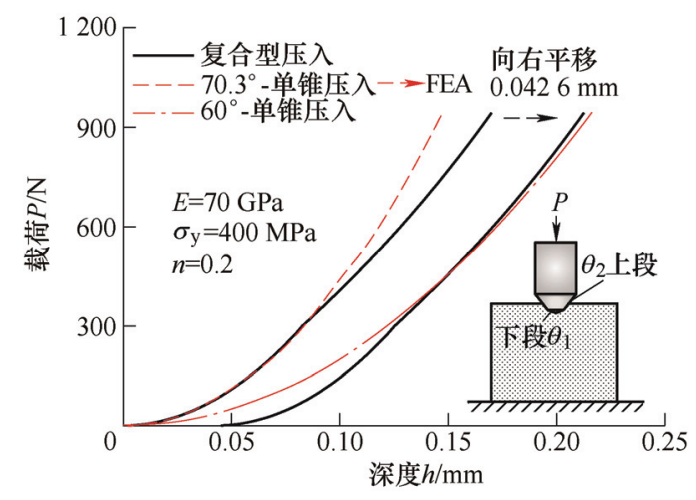

图 9 双雉复合型压入与单雉压入曲线的对比

进一步针对屈服应力 $\sigma_{\mathrm{y}} \in[100,600] \mathrm{MPa}$ 、硬化指 数 $n \in[0.1,0.4]$ 时的铝合金材料进行广泛的模拟, 发现 结果均与图 9 的现象相符。通过按照 Kick 律回归双雉 复合型压入曲线的两个阶段得到相应的加载曲率

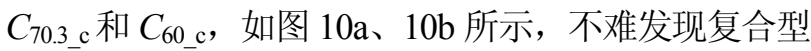
压入的第一段加载曲率 $C_{70.3 \mathrm{c}}$ 与 $70.3^{\circ}$ 单雉压入加载 曲率 $C_{70.3 \mathrm{~s}}$ 完全一致; 而第二段加载曲线平移至符合 Kick 律后, 其加载曲率 $C_{70.3} \mathrm{c}$ 与 $60^{\circ}$ 单雉压入加载曲 率 $C_{60 \mathrm{~s}}$ 可建立近似的比例关系, 即 $C_{60} \mathrm{c}=1.04 C_{60} \mathrm{~s}$ 。

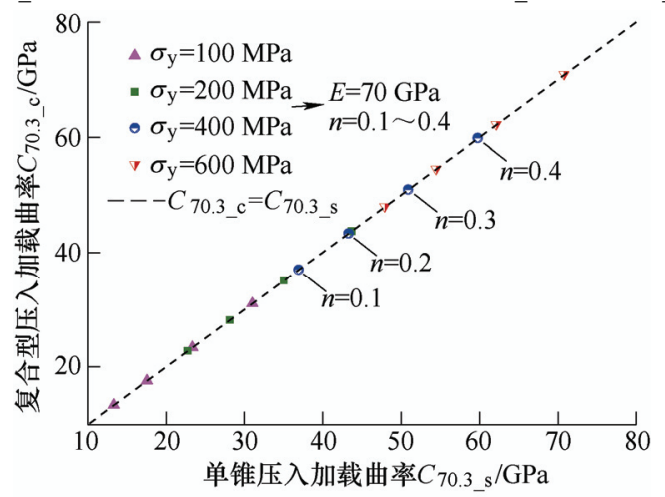

(a) $\theta=70.3^{\circ}$

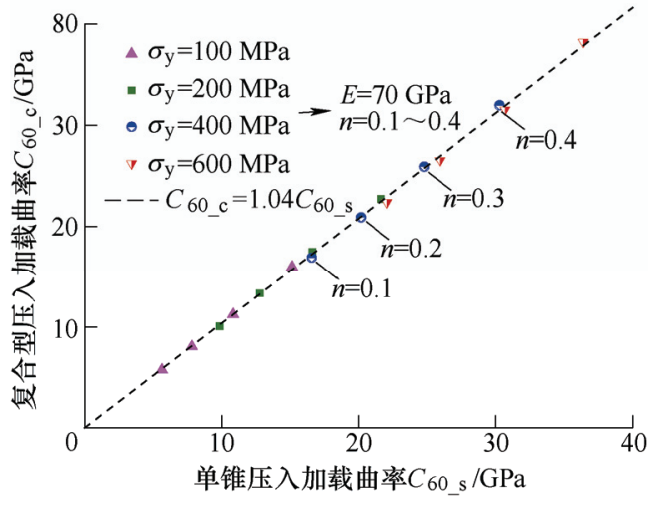

(b) $\theta=60^{\circ}$

图 10 复合型双雉压入与单雉压入加载曲率的对比 
通过复合型双雉压头单次压入材料表面, 结合 复合型压入与单雉压入的简单关联及单锥压入理论 模型, 同样可实现材料单轴力学性能参数的获取, 称之为复合型双雉压入方法(Composite dual-conical indentation method, CDIM)。

考虑加工精度限制使得压头初始尖端总存在微 小的针形, 且随着压入次数的增加, 压头累积变形 与磨损将导致其尖端的进一步针化。因此考虑压头 尖端钝化的影响十分必要, 参考通常的做法可将压 头钝化轮廓考虑成不同曲率半径的圆弧进行分 析 $^{[22]}$ 。对任选铝合金材料, 图 11a 给出了不同程度 钝化后载荷-深度曲线与无针化的理想载荷-深度曲 线之间对比。不难看出, 当压尖钝化曲率半径在 20 $\mu \mathrm{m}$ 以内变化时, 复合型双雉压入的两阶段载荷-深 度曲线始终保持着良好的重合性。而由图 $11 \mathrm{~b}$ 给出 两阶段量化的加载曲率可知, 当压尖钝化曲率半径 在 $20 \mu \mathrm{m}$ 以内时, 随着压头钝化曲率半径的增加, 上下两段的加载曲率均逐渐增大, 且导致下段加载

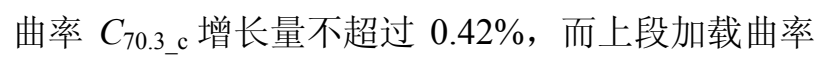
$C_{60 \_c}$ 增长量不超过 $1.8 \%$, 即影响的确较小。由此可 见, 控制好压头加工精度和注意在使用过程中定期 对压头轮廓进行检查即可有效避免压头钝化带来的 不利影响。

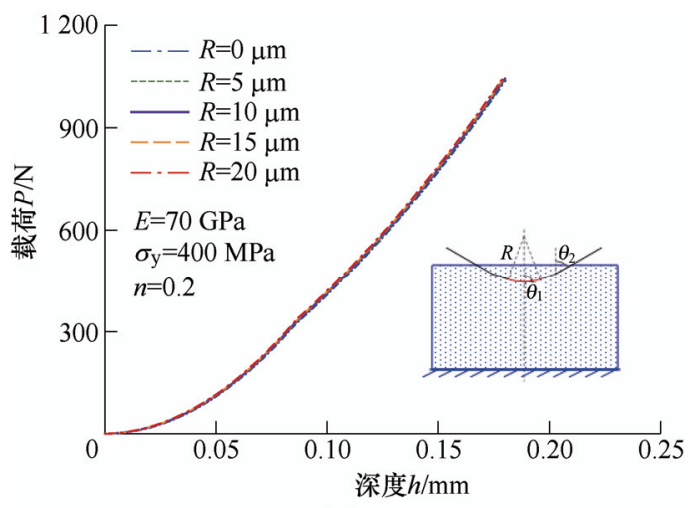

(a) 复合型压入 $P-h$ 曲线

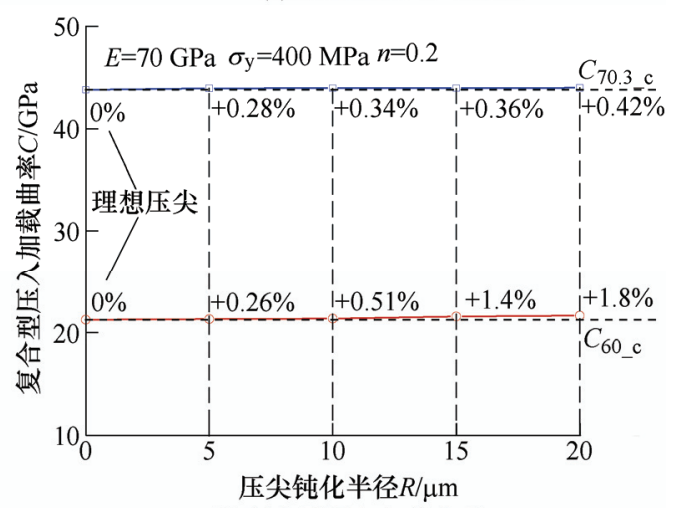

(b) 复合型压入加载曲率

图 11 尖端钝化半径对载荷-深度曲线及加载曲率的影响

此外, 为探究下段圆雉高度 $H$ 对上段圆雉压入
加载曲率的影响。仍以任选材料为例, 得到不同 $H$ 下载荷-深度曲线及上段压头压入加载曲率的对比 情况分别如图 12a、12b 所示。为保证下段圆雉压入 具有足够的载荷-深度数据以便准确获取 $C_{70.3 \mathrm{c}}$, 将 $H$ 设置为 $60 \sim 100 \mu \mathrm{m}$ 。由图 12a 可知, 当 $H$ 在 $60 \sim$ $100 \mu \mathrm{m}$ 时, 下段圆雉压入的载荷-深度曲线维持不 变, 而上段压入的载荷-深度曲线呈互相平行趋势, 且平移处理后的各种 $H$ 高度对应的上段载荷-深度 曲线基本重合。从图 $12 \mathrm{~b}$ 可看出, 下段圆雉高度 $H$ 对上段圆雉压入加载曲率的影响在 $1.1 \%$ 以内。

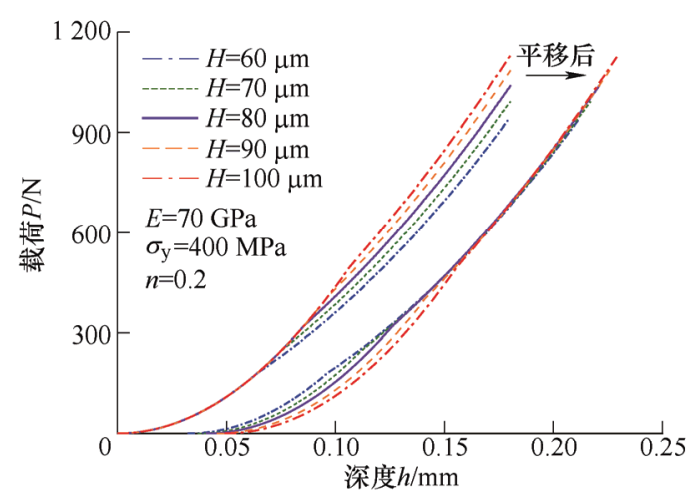

(a) 复合型压入 $P-h$ 曲线

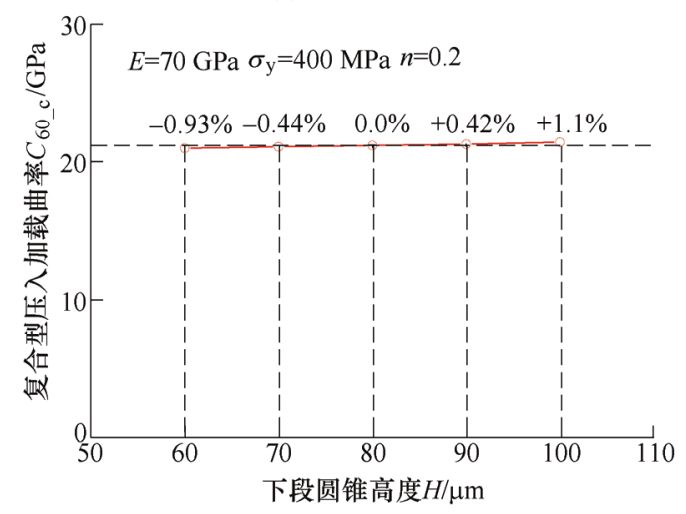

(b) 复合型压入加载曲率

图 12 下段圆雉高度 $H$ 对载荷-深度曲线及加载曲率的影响

\section{4 复合型双雉压入测试}

为了验证 CDIM 的实用性, 选用 5052-H112、 6061-T652 及 7075-T651 三类铝合金材料小试样 $(20 \mathrm{~mm} \times 20 \mathrm{~mm} \times 10 \mathrm{~mm})$ 进行了复合型双雉压入试 验。压头为下段为 $70.3^{\circ}$ (高度约为 $80 \mu \mathrm{m}$ ), 上段为 $60^{\circ}$ 的复合型双锥压头, 被测铝合金材料表面经过 抛光处理。试验采用位移控制, 加载速率 $0.003 \mathrm{~mm} / \mathrm{s}$, 最大压深为 $0.17 \mathrm{~mm}$ 。为了保证测试结 果的可靠性, 同一材料试样表面不同位置重复压入 3 次, 得到的试验曲线如图 13 所示。分别将下段 $70.3^{\circ}$ 原始压入曲线与上段 $60^{\circ}$ 压入曲线平移后的 结果依据 Kick 定律按照图 4 的 $h-P^{1 / 2}$ 正比例关系拟 
合得到加载曲率, 并取平均值, 具体结果列于表 2 。

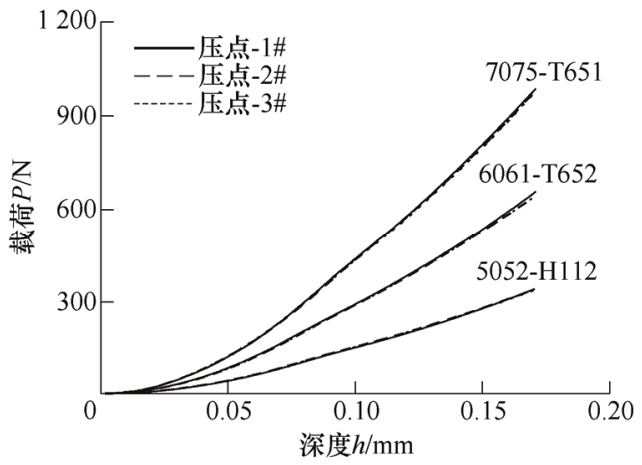

图 13 三种铝合金复合型压入试验曲线

由图 13 可知, 同一材料不同压点之间的分散性 较低。图 $14 \mathrm{a} \sim 14 \mathrm{c}$ 分别给出了 3 种铝合金在复合型 双雉压入加载下的载荷-深度响应与有限元计算得 到的单雉压入 $\left(60^{\circ}\right.$ 和 $\left.70.3^{\circ}\right)$ 载荷-深度曲线的对比 关系。由图 14 可以看出, 复合型压入试验曲线呈现

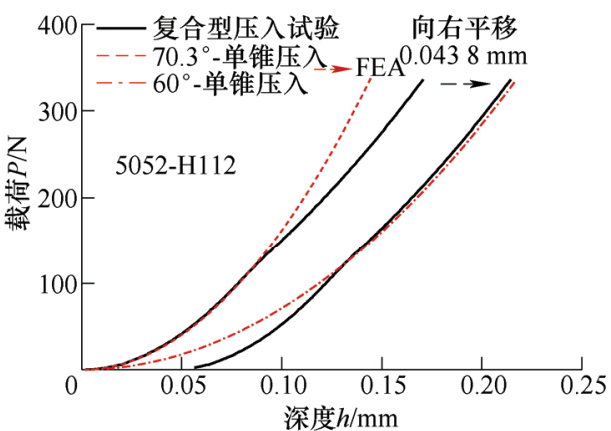

(a) $5052-\mathrm{H} 112$

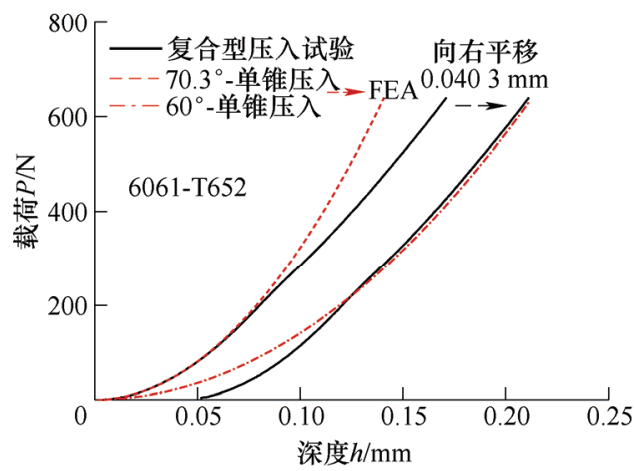

(b) 6061-T652

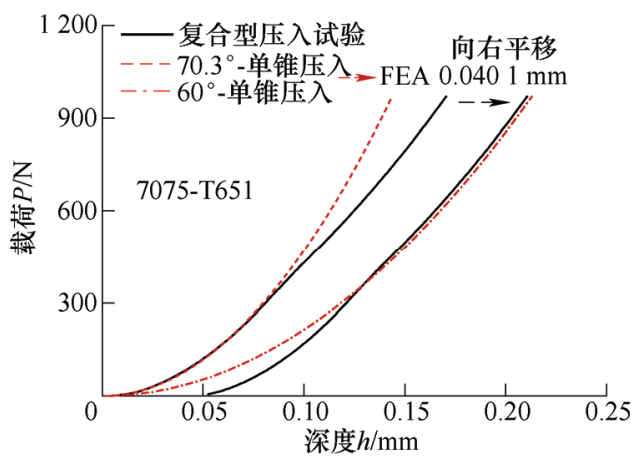

(c) 7075-T651

图 14 复合型压入试验曲线与单雉压入 FEA 计算曲线对比
与图 9 类似的现象, 且均与 FEA 模拟曲线吻合较好, 这也说明上段雉角完全进入材料后对下段雉角压入 的 Kick 律影响较小, 下段压入曲线所对应的功或能 的增量几近是按上段压头单独压入时的方式增长。

根据表 2 给出的各材料压点加载曲率值 $C_{70.3} \mathrm{c}$ 与 $C_{60} \mathrm{c}$, 分别按照图 $10 \mathrm{a}$ 和图 $10 \mathrm{~b}$ 所示关系转换成 $C_{70.3 \mathrm{~s}}$ 及 $C_{60 \_\mathrm{s}}$ (即 $C_{70.3 \mathrm{~s}}=C_{70.3 \mathrm{c}}, C_{60 \mathrm{~s}}=C_{60} \mathrm{c} / 1.04$ ), 将它们的平均值代入式(22)即可求得被压材料塑性 参数, 结合已知铝合金弹性模量 $E$ 约为 $70 \mathrm{GPa}$, 最 终得到了图 15 所示的压入应力-应变关系曲线同拉 伸试验曲线的对比情况。可以看出, 图 15 中虚线表 示的雉形压入结果与实线表示的拉伸曲线较为接 近，最大误差不超过 7\%。

表 2 圆雉压入试验曲线的加载曲率值

\begin{tabular}{cccccc}
\hline 材料 & 加载曲率 & $1 \#$ & $2 \#$ & $3 \#$ & 平均值 \\
\hline $5052-\mathrm{H} 112$ & & 16314 & 16120 & 16071 & 16169 \\
$6061-\mathrm{T} 652$ & $C_{70.3 \_c}$ & 32822 & 32398 & 32463 & 32561 \\
$7075-\mathrm{T} 651$ & & 47882 & 46985 & 47220 & 47362 \\
\hline $5052-\mathrm{H} 112$ & & 7523 & 7457 & 7435 & 7472 \\
$6061-\mathrm{T} 652$ & $C_{60 \_c}$ & 14871 & 14705 & 14769 & 14782 \\
$7075-\mathrm{T} 651$ & & 22529 & 22238 & 22284 & 22350 \\
\hline
\end{tabular}

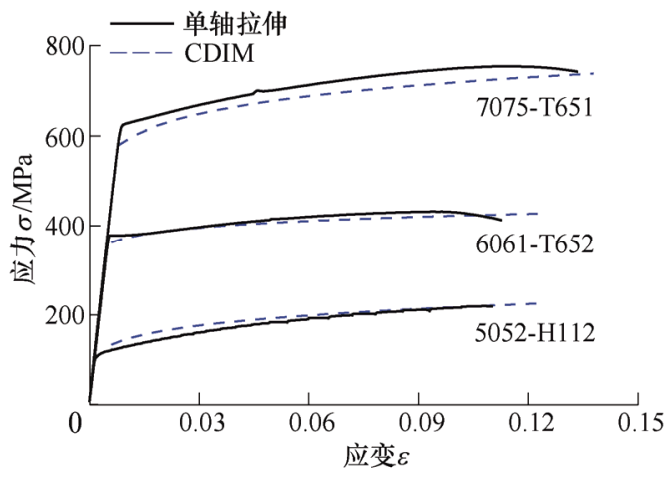

图 15 压入应力-应变曲线与拉伸结果的对比

\section{5 结论}

(1) 本文基于能量等效原理导出了雉压入弹塑 性模型, 该模型参数少且给出了预测材料塑性参数 的显式表达式。借助有限元分析在铝合金材料范围 内，验证了模型正向、反向预测的准确性。

(2) 通过设计集两种雉角于一体的复合型压 头, 利用有限元计算确定了复合型双雉压入与单雉 分别压入之间的简单关联, 并据此提出了复合型双 雉压入方法。

(3) 针对 3 种常用铝合金材料, 采用所提复合 
型双雉压入方法进行了测试研究, 结果表明该方法 预测的应力-应变关系与拉伸结果较为一致。该方法 不仅对铝合金适用，还将进一步应用于同样满足各 向同性，等向强化的钢材、钛合金、聚合物等的力 学性能获取。

\section{参 考 文 献}

[1] BUCAILLE J L, STAUSS S, FELDER E, et al, Determination of plastic properties of metals by instrumented indentation using different sharp indenters [J]. Acta Materialia, 2003 , 51(6): 1663-1678.

[2] DAO M, CHOLLACOOP N, VAN VLIET K J, et al. Computational modeling of the forward and reverse problems in instrumented sharp indentation[J]. Acta Materialia, 2001, 49(19): 3899-3918.

[3] CAO Y P, QIAN X Q, LU J, et al. An energy-based method to extract plastic properties of metal materials from conical indentation tests[J]. Journal of Materials Research, 2005, 20(5): 1194-1206.

[4] OGASAWARA N, CHIBA N, CHEN X. Representative strain of indentation analysis[J]. Journal of Materials Research, 2005, 20(8): 2225-2234.

[5] BRANCH N A, SUBHASH G, ARAKERE N K, et al. Material-dependent representative plastic strain for the prediction of indentation hardness[J]. Acta Materialia, 2010, 58(19): 6487-6494.

[6] 吉喆, 高二庆, 沈承金, 等. 预加载条件下钛合金雉形 压痕代表应变的确定 [J]. 塑性工程学报, 2019, 26(4): 213-219.

JI Zhe , GAO Erqing, SHEN Chengjin, et al. Determination of representative strain of titanium alloy induced by sharp indentation under preload condition[J]. Journal of Plasticity Engineering, 2019, 26(4): 213-219.

[7] THO K K, SWADDIWUDHIPONG S, LIU Z S, et al. Uniqueness of reverse analysis from conical indentation tests[J]. Journal of Materials Research, 2004, 19(8): 2498-2502.

[8] 姚博, 蔡力勋, 包陈. 基于锥形压入的材料力学性能测 试方法研究 [J]. 航空学报, 2013，34(8): 1874-1883. YAO Bo, CAI Lixun, BAO Chen. Research on acquisition of mechanical properties of materials based on conical indentation[J]. Acta Aeronautica et Astronautica Sinica, 2013, 34(8): 1874-1883.

[9] PÖHL F. Determination of unique plastic properties from sharp indentation[J]. International Journal of Solids and
Structures, 2019, 171: 174-180.

[10] 石新正, 陈伟, 陈平, 等. 金属材料弹塑性参数仪器化 聚集式 Vickers 压入识别方法[J]. 中国有色金属学报, 2019, 29(11): 2582-2593.

SHI Xinzheng, CHEN Wei, CHEN Ping, et al. Method for determining elastic-plastic parameters of metals by instrumented gathered indentation with Vickers indenter[J]. The Chinese Journal of Nonferrous Metals, 2019, 29(11): 2582-2593.

[11] PEYROT I, BOUCHARD P O, GHISLENI R, et al. Determination of plastic properties of metals by instrumented indentation using a stochastic optimization algorithm[J]. Journal of Materials Research, 2009, 24(3): 936-947.

[12] HAJ-ALI R, KIM H K, KOH S W, et al. Nonlinear constitutive models from nanoindentation tests using artificial neural networks[J]. International Journal of Plasticity, 2008, 24(3): 371-396.

[13] 陈辉, 蔡力勋, 包陈. 双锥度压入的 FAT 迭代法获取材 料的力学性能[J]. 核动力工程, 2015, 36(5): 101-104. CHEN Hui, CAI Lixun, BAO Chen. FAT iterative method based on dual conical indentation to obtain properties of materials[J]. Nuclear Power Engineering, 2015，36(5): 101-104

[14] HOSSEINZADEH A R , MAHMOUDI A H. Determination of mechanical properties using sharp macro-indentation method and genetic algorithm $[\mathrm{J}]$. Mechanics of Materials, 2017, 114: 57-68.

[15] CHEN Hui, CAI Lixun. Theoretical model for predicting uniaxial stress-strain relation by dual conical indentation based on equivalent energy principle[J]. Acta Materialia, 2016, 121: 181-189.

[16] CHEN Hui, CAI Lixun. Unified elastoplastic model based on a strain energy equivalence principle[J]. Applied Mathematical Modelling, 2017, 52: 664-671.

[17] SNEDDON I N. The relation between load and penetration in the axisymmetric Boussinesq problem for a punch of arbitrary profile[J]. International Journal of Engineering Science, 1965，3(1): 47-57.

[18] 张泰华. 微/纳米力学测试技术: 仪器化压入的测量、 分析、应用及其标准化 [M]. 北京: 科学出版社, 2013 . ZHANG Taihua. Micro/Nano mechanical testing technology: Measurement, analysis, application and standardization of instrumented indentation[M]. Beijing: Science Press, 2013.

[19] 徐连勇, 张舒婷, 荆洪阳, 等. $\mathrm{Ag}-\mathrm{GNSs} / \mathrm{SnAgCu}$ 钎料 
纳米压痕变形行为研究 [J]. 机械工程学报, 2018, 54(8):

151-156.

XU Lianyong, ZHANG Shuting, JING Hongyang, et al. Research on the deformation behavior of Ag-GNSs/ $\mathrm{SnAgCu}$ solders during nanoindentation tests[J]. Journal of Mechanical Engineering, 2018，54(8): 151-156.

[20] JOHNSON K L. Contact mechanics[M]. Cambridge: Cambridge University Press, 1985.

[21] HYUN H C, KIM M, LEE J H, et al. A dual conical indentation technique based on FEA solutions for property evaluation[J]. Mechanics of Materials, 2011, 43(6): 313-331.

[22] 张赜文, 余家欣, 钱林茂. 压头曲率半径对单晶硅径向
纳动损伤的影响 $[\mathrm{J}]$. 机械工程学报, 2010, 46(9): 107-112.

ZHANG Zewen, YU Jiaxin, QIAN Linmao. Effect of curvature radius of indenter on the radial nanofretting damage of monocrystal silicon[J]. Journal of Mechanical Engineering, 2010, 46(9): 107-112.

作者简介: 陈辉, 男, 1990 年出生, 院聘副教授。主要研究方向为材料 测试理论与方法。

E-mail: chen_hui5352@163.com

彭晖(通信作者), 男, 1976 年出生, 教授, 博士研究生导师。主要研究 方向为新材料与新型结构的力学与耐久性能。

E-mail: huipeng@esust.edu.cn

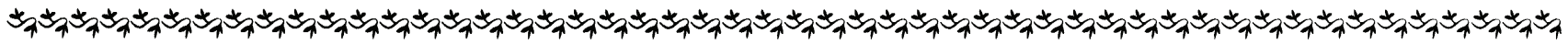

(上接第 78 页)

计算机学报, 1997, 20(4): 381-384.

WANG Xuemei, WANG Yihe. The combination of simulated annealing and genetic algorithms[J]. Chinese Journal of Computers, 1997, 20(4): 381-384.

[26] 杨源杰, 黄道. 人工神经网络算法研究及应用[J]. 华东 理工大学学报, 2002, 28(5): 115-118.

YANG Yuanjie, HUANG Dao. Research and applications of artificial neural networks[J]. Journal of East China University of Science and Technology, 2002, 28(5): 115-118.

[27] BARLAT F, YOON S Y, LEE S Y, et al. Distortional plasticity framework with application to advanced high strength steel[J]. International Journal of Solids and Structures, 2020，202: 947-962.

作者简介: 付佳伟, 男, 1983 年出生, 博士, 副教授, 硕士研究生导 师。主要研究方向为新型材料力学属性反问题求解理论、数值模拟和 测试技术。

E-mail: jiawei.fu@nwpu.edu.cn 齐乐华(通信作者), 女, 1957 年出生, 博士, 教授, 博士研究生导师。 主要研究方向为轻质合金基复合材料成形工艺及理论。

E-mail: qilehua@nwpu.edu.cn 